\title{
New strain gauge-based torque reference chain offering smallest measurement uncertainties
}

\author{
Dr. André Schäfer \\ Hottinger Baldwin Messtechnik GmbH (HBM), Darmstadt, Germany
}

\begin{abstract}
New measurement approaches for mechanical quantities based on strain gauge technology offer one feature above all else: the possibility to realize smallest measurement uncertainties. This is particularly important for metrological infrastructure, i.e. realization of metrological traceability chain to support applications in aerospace or in automotive industry. Achievements toward smaller measurement uncertainties are shown by realization of a novel torque reference sensor $\mathrm{TN}$ with improved usability for inter-laboratory comparisons in combination with a surprisingly compact precision amplifier called Quantum MX238B.
\end{abstract}

\section{Introduction}

Regarding high precision measuring chains, the choice of strain gauge-based torque reference chain offers smallest measurement uncertainties. Therefore, they are so widespread in metrology use and in the traceability chains of mechanical quantities.

\section{Reference transducers}

Regarding the reference transducer, to be able to benefit from the advantages offered by a monolithic design one must choose a shaft-type torque transducer, which requires an "open" design, meaning that the strain gauge cannot be hermetically sealed. The strain gauge is therefore dependent on environmental conditions. As torque transfer standards are meant for inter-laboratory comparisons, its usability under changing conditions is of major importance and therefore critical to duration and success of the procedure. Therefore, a detailed analysis of the application conditions of "travel standards" during inter-laboratory comparisons resulted in ideas that have been implemented in the new "TN" torque transfer standard.

By the principle used in the transducer, beside of temperature, also humidity needs to be carefully considered as a significant source of uncertainty. Humidity deserves special 
attention, as "open" strain-gauge transducer designs are known to be influenced by humidity due to the hygroscopic behavior of carrier of the foil-type S.G.

Figure 1 and 2 show the outline of the new transducer and its new carrying case.

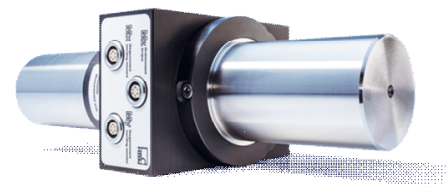

Fig. 1: TN torque transfer standard

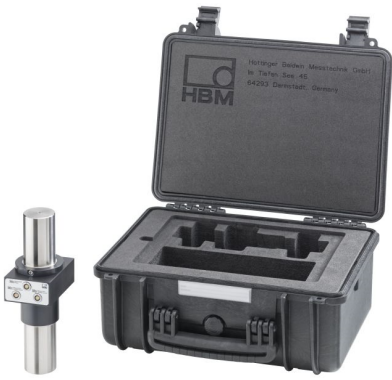

Fig. 2: New carrying case with sealing lip

Because of the special application requirements, however, some frame conditions had to be considered. Two main design choices for a reference torque transducer shown in Figure 3 and 4.
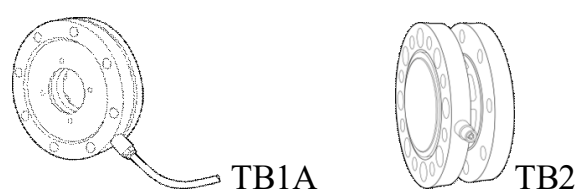

Fig. 3: Different types of flange torque transducers

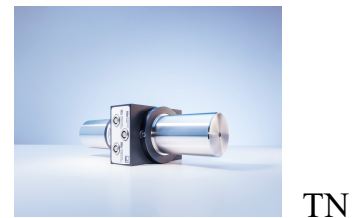

Fig. 4: Shaft type torque transducer

\section{High precision amplifiers}

For the other part of the reference measurement chain, the high precision amplifier used for reading out the data, due to latest developments very high requirements can be achieved. Although very compact and offering still two independent channels the Quantum MX238B uses an active temperature compensation shows nearly no dependency on temperature, whether is about span or zero point. Finally, working with a 24-bit A/D converter, the compact instrument reaches an accuracy class of $25 \mathrm{ppm}$. Most important to the customer however should be, the patented background calibration ensuring processing measurement without any interruption, even if internal calibration is processed. 
HBMs Flagship DMP41 is the most precise amplifier in the world for strain gauge-based measurements. This is another choice for completing the measuring chains, preferably for primary applications.

The DMP series has been continuously developed by HBM for more than 30 years. It offers outstanding long-term stability and ultra-modern, user friendly functions, such as touch screen operation.

DMP41 is available as a desk top device and is also available version with cabinet housing. Fig. 5 shows DMP41 in a version as rack mounted (cabinet housing).

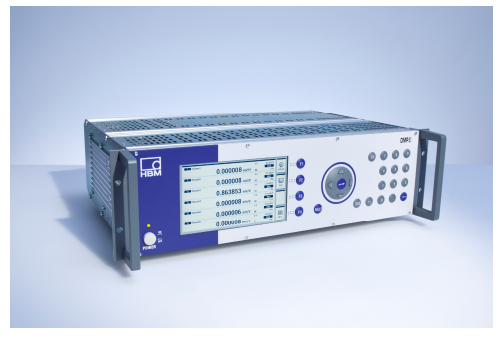

Fig. 5: DMP41, version with cabinet housing

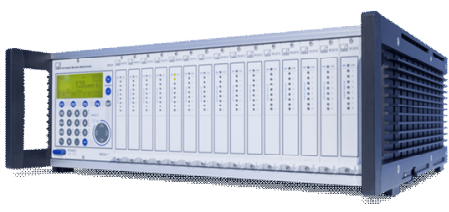

Fig. 6: ML38B of MGCplus System

The precision amplifier supports simultaneous measurement with multiple channels. Moreover, DMP41 offers even higher noise immunity. Its state-of-the-art interface allows integration with modern laboratory network environments in many ways. Metrology institutes can trust that they get maximum precision.

Minimal variations at high static loads are often measured in research and science as well as in the manufacture of high-precision elements. The DMP41 precision amplifier shows its strengths with high-precision comparison measurements of mechanical quantities. Many national test institutes, including the German National Metrology Institute (PTB), use DMP series instruments for their own measurements; however, the DMP41's technical benefits such as accuracy, high resolution and long-term stability are also important in the development, production and quality assurance of strain gauge transducers. 
Electronics are also influenced by humidity and, in terms of precision electronics, stability plays an important role. Therefore, the so-called "climate safety of electronic assemblies" has been a research topic already [5]. It was found that, apart from incorrect measurement results, error images such as electrochemical migration, leakage currents, flashover, solder joint load, and corrosion may occur. In [6] the stability of BN100A under humidity and/or temperature change has been verified, while DMP40, the predecessor of DMP41, still seemed to show a slight linear trend line with increasing humidity or temperature. This is also the reason why we are paying close attention to this point: in the selection of electronic components, but also in such factors as the instruments' inner space and even in the housing design, such as the general use of metal housings [7]. It was also pointed out by other sources [8], that due to necessity of the condensation of water at the inside wall the cabinet the use of metal housing is essential for building up precision amplifiers

\section{Meeting the requirements for inter-laboratory comparisons}

As pointed out before, the practical problem during inter-laboratory comparisons is not only the use in the lab but also the transport route from one laboratory to another. Problems with the use of transfer standards may occur on your "journey" for comparison measurements from country to country, for instance, during the transport in the cargo space of intercontinental flights, where the devices often are stored at about $5^{\circ} \mathrm{C}$ for many hours and, after the arrival, for example, in tropical countries immediately exposed to $30^{\circ} \mathrm{C}$ for hours of waiting on the runway/airfield of an airport.

Therefore, the usefulness of the new carrying case with a special sealing lip is not to be underestimated. It effectively preserves the "microclimate" around the transducer. To use this effect, it is recommended to directly pack the transducer in the laboratory environment and to close the carrying case tightly, so that one "takes along" the ambient air of the lab. When arriving at the "next" laboratory in the daisy chain of the "inter-laboratory comparison", it is essential to wait for an appropriate time, until a new balance in terms of humidity and temperature is reached. Some NMIs have expressed that they need a better device coping with $40 \pm 2 \%$ relative humidity with a change of a few ppm only. Other institutes even demand similar stability for much bigger changes [9]. It can thus be assumed that the described measures will significantly improve the suitability for the application.

\section{Conclusion}


The paper demonstrated that the complete measurement chain plays in the 200 to $300 \mathrm{ppm}$ uncertainty class, what is a new achievement compared to everything made before and can be considered a substantial contribution to ensure better traceability of the quantity torque.

TN transducer is a top-of-the-line model for torque reference applications [10]; [11]. It can be used as a transfer standard for comparison and for traceability in torque calibration systems, as well as a reference sensor in calibration laboratories using the reference principle. [12]; [13]; [14]. Under the given design choices, the improvements for the sensor itself and in conjunction with a new carrying case provide both, good mechanical protection as well as good insulation from different environmental conditions.

Generally, a reduction in moisture sensitivity due to the use of a less hygroscopic material also allows shortening the above-mentioned time of enough "balancing" before starting the measurement. The necessity has been repeatedly noted in scientific articles of the PTB and other NMIs [15]; [16]. More recent results of humidity investigations are published in [17] as well.

\section{References}

[1] Schäfer, A. "TN torque transfer standard with improved usability for interlaboratory comparations", Proceedings of IMEKO XXII World Congress, September 2018, Belfast NI, UK

[2] Röske, D., Key comparisons in the field of torque measurement, 19th IMEKO TC3 on Force, Mass and Torque: "Theory and Application in Laboratories and Industries", 2005, Cairo, Egypt.

[3] Brüge, A. Simplified measurements of the humidity coefficient of torque transducers in calibration laboratories, ACTA IMEKO June 2014, Volume 3, Number 2, 32 - 38, www.imeko.org

[4] Schäck, M, "Improving the Characteristics of a High-Precision Measuring Amplifier by a Powerful Digital Signal Processing”, Proceedings of IMEKO XXII World Congress, September 2018, Belfast NI, UK

[5] Ring, K. Klimasicherheit von elektronischen Baugruppen (engl. transl.: Climate safety of electronic assemblies), ZVE Fraunhofer Institut für Zuverlässigkeit \& Mikrointegration, Berlin, 2007 
[6] Käs, G. Qualität und Zuverlässigkeit elektronischer Bauelemente und Systeme, R. Oldenburg publishing, Vienna, 1983, ISBN-3-486-2735-5

[7] Khaled, K.M.; Röske, D. at al. Humidity and temperature effects on torque transducers, bridge calibration unit and amplifiers, Measurement 74 (2015) p. 3142, Elsevier B.V. Publishing house, N.Y.; U.S.A.

[8] Drung, D. at al. Improvements in the field of traceable high-accuracy measurement and generation of small electrical currents, Presentation of Helmholtz award winners, PTB WG 2.61, Bad Honnef, Germany, May 2018

[9] Röske, D. Final report on the torque key comparison CCM.T-K1: Measurand torque: 100 Nm, 500 Nm, 1000 Nm, Metrologia, 46 (2009), Tech. Suppl., 07006.

[10] Schäfer, A. The Ultra-Precision Instrument DMP41 -First Experiences \& Appropriate Filter settings, TC3 Conference, 2014, Cape Town, South Africa

[11] Schäfer, A. DMP41-A new Chapter of Ultra-Precision Instrument for Strain Gauge Transducers XX IMEKO World Congr. 2012, Busan, Rep. of Korea

[12] Schäfer, A. Answers to the need of higher orders of magnitude for pressure, force \& torque measurement IEEE I2MTC Conference Graz, Austria, March 2012

[13]Hohmann, P.: Schäfer, A. , Combined Calibration of Torque and Force in a 3 in 1 Calibration unit, “APMF 2000”, Asia-Pacific Symposium on Measurement of Mass, Force \& Torque, pp. 204, Tsukuba, Japan, 2000

[14]Hoffmann, K. ; An Introduction to Measurements using Strain Gauges , Hottinger Baldwin Messtechnik GmbH, Germany, 1989, pp. 145

[15]Brüge, A., Fast Torque Calibrations Using Continuous Procedures, 18th Conference on Force, Mass and Torque, 24-26, 2002, Celle, Germany

[16] Sanponpute, T. ; Arksonnarong, N. Temperature \& humidity dependence on stability of torque device IMEKO TC3, TC5, TC22 Conf., 2014, Cape Town, South Africa

[17] Khaled, K.M.; Röske, D. at al. The influence of temperature and humidity on the sensitivity of torque transducers, Measurement 94 (2016) p. 186-200, Elsevier B.V. Publishing house, N.Y.; U.S.A. 